\title{
Is the Patient Getting Worse?
}

\author{
Kuldev Singh*
}

Glaucoma Service, Stanford University, USA

\begin{abstract}
Glaucoma care today is often simplified into all or nothing terms with the assumption that if the patient's intraocular pressure (IOP) is above a certain level, glaucomatous disease will progress and when the IOP is lowered to below a threshold, no further progression will occur. This dogma is largely the result of limitations in the resolution of tools currently available to judge progression. Glaucoma is a neurodegenerative disease and, as with all degenerative diseases, progression continues to occur, albeit at slower rates, with appropriate treatment. In the future, as our tools to assess structural and functional optic nerve change become more precise, we will no longer think of glaucoma as being stable or progressive but rather speak in terms of rates of progression.
\end{abstract}

There was a time when primary open-angle glaucoma was considered a disease of increased intraocular pressure (IOP). If one's IOP was above the $95^{\text {th }}$ percentile upper limit of normal, $(21 \mathrm{~mm} \mathrm{Hg}$ in several Caucasian populations throughout the world) one would make the diagnosis of glaucoma. If IOP was lowered in such a patient to less than $21 \mathrm{~mm} \mathrm{Hg}$, the patient would then be considered to have controlled glaucoma. If the IOP remained greater than 21 $\mathrm{mm} \mathrm{Hg}$ despite treatment, one would expect the patient to get worse (i.e., show progressive visual deterioration).

Several large prevalence surveys of glaucoma showed us that the relationship between IOP and primary open-angle glaucoma was not so simple. One such study was the Baltimore Eye Survey, conducted in East Baltimore, MD in the neighborhoods adjacent to Johns Hopkins Hospital. This study of over 5,000 individuals showed that approximately half of all individuals found to have open-angle glaucoma had an initial IOP reading of less than $21 \mathrm{~mm} \mathrm{Hg}[1,2]$. Even with three IOP readings, $20 \%$ of those with glaucomatous disease were found to not have a single reading above $21 \mathrm{~mm} \mathrm{Hg}$. Further studies in North America and other regions of the world confirmed that the IOPglaucomatous disease relationship is continuous with no arbitrary IOP cutoff below and above which one's disease will be stable or progressive respectively [3-7]. One such study in a Japanese population showed that even the $95^{\text {th }}$ percentile upper limit of IOP is not consistent across populations [4]. Both normal and glaucomatous individuals in Japan have lower IOP's, on average, than similarly normal and diseased individuals in the United States.

A series of multicenter randomized clinical trials conducted in North America and Europe over the past 20 years have shed more light on the IOP-primary open-angle glaucoma relationship [8-11]. The target goals for IOP lowering have varied between these studies with most aiming for a percentage reduction in IOP from baseline and one, the Advanced Glaucoma Intervention Study, setting an arbitrary number $(18 \mathrm{~mm} \mathrm{Hg})$ as the target for successful

*Address correspondence to this author at the Glaucoma Service, Stanford University, USA; E-mail: kuldev.singh@stanford.edu therapy. Glaucoma practice has evolved to replace the global target of $21 \mathrm{~mm} \mathrm{Hg}$ with individualized target goals for patients depending upon disease severity and risk factors for vision loss. Most acknowledge that these target goals can change depending upon numerous factors including disease course, life expectancy and risks and benefits of the various therapies needed to achieve these goals.

Nevertheless, the fundamental principle that glaucoma will progress if the IOP is above a certain level and stabilize if it is below that level remains pervasive in practice today. We live in a binary world when it comes to glaucoma assessment and decision making. When glaucoma practitioners discuss patients with each other today, the terms they use include "above target" and "below target" when it comes to IOP. With regard to functional and structural assessment of the optic nerve, the commonly used terms include "stable" versus "progressive". The twenty first century glaucoma dogma of glaucoma assessment and management can be summed up by the following: if IOP is above a target, glaucoma will worsen and if it is below target, glaucoma progression will be halted. Post-hoc analyses from randomized clinical trials that had been designed to answer other issues have been created to support this dogma [8]. There are some who believe that IOP lowering alone can completely halt glaucoma progression. Given that primary open-angle glaucoma is a chronic multifactorial neurodegenerative disease, the proposal that it can completely be arrested simply by impacting one risk factor is surprising, to say the least.

One reason that the present day dogma exists undoubtedly relates to the limitations of the tools that we currently have to assess glaucomatous disease including progression. For example, there are some patients with primary open-angle glaucoma based upon serial optic nerve examination who continues to have "normal" tests of optic nerve function with white-on-white automated perimetry. With regard to progression, there are eyes that are losing ganglion cells and axons over periods of time during which there are no statistical changes noted on white-on-white perimetric testing. The reverse may be true as well. There are circumstances when structural optic nerve assessment fails to 
reveal statistical change while vision continues to deteriorate. The lack of resolution of the tools to confirm disease and disease progression in many circumstances when such disease and disease progression is present has significantly contributed to the binary glaucoma world in which we currently live.

Another enabling factor for this binary dogma may exist is that it simplifies the physician-patient encounter. It allows one to quickly reassure patients who have IOPs below target and are not showing progression while increasing therapy in those where IOP is above target or structural and/or functional optic nerve change has been noted.

The tools we possess for assessing structural and functional damage related to glaucoma including progression will improve dramatically in the coming years. In the not too distant future, a practitioner will be able to assess the number and function of living retinal ganglion cells in the clinic setting. Imaging devices will improve in resolution such that rates of change will be more precise. Perimetric testing will be able to pick up smaller increments of change. All of these advances will revolutionize how we view glaucoma as a disease. Most notably, we will do away with the binary dogma that has plagued glaucoma care for the past century. We will correctly recognize that glaucoma, like all chronic diseases, cannot be halted, and only slowed. It is well known that aging has been estimated to result in loss of approximately 5,000-10,000 retinal ganglion cells per year [12]. Other non IOP related risk factors will undoubtedly also be found to be important.

The major impact of such improved resolution of diagnostic tools for glaucoma care will be the focus on rates rather than on binary outcomes. It will be acknowledged that everyone is getting worse, some faster than others. It will not be sufficient to tell patients that their IOP is below target and thus they are not progressing or that additional therapy is necessary to lower IOP until they reach a lower level where they will no longer show such progression. When rates of change become readily available, this information will be shared with patients such that they better understand the pathophysiology and natural history of their glaucomatous disease.

The irony in all of the advances that are to come is that they will do more to remind us that glaucoma care is art rather than making treatment more scientific. Therapy will not be advanced because a patient shows statistical progression on a visual field test but rather because the rates of progression are deemed unacceptable given the patient's severity of disease, overall health, age, risks and benefits of various treatment options and patient willingness to accept these risk-benefits trades-off. Unlike care in the present era of binary dogma, not all progression will have to be met with additional treatment because all patients will be deemed to be continually progressing, albeit at different rates.

\section{REFERENCES}

[1] Tielsch JM, Katz J, Singh K, et al. A population-based evaluation of glaucoma screening: The Baltimore Eye Survey. Am J Epidemiol 1991; 134: 1102-10.

[2] Sommer A, Tielsch JM, Katz J, et al. Relationship between intraocular pressure and primary open angle glaucoma among white and black Americans: the Baltimore eye surgery. Arch Ophthalmol 1991; 109: 1090-95.

[3] Banks JLK, Perkins ES, Tsolakis S, Wright JE. Bedford Glaucoma Survey. Br Med J 1968; 30: 791-79.

[4] Shiose Y, Kitazawa Y, Tsukahara S. The epidemiology of glaucoma in japan: a nationwide glaucoma survey. Jpn J Ophthalmol 1991; 35(2): 133-55.

[5] Munoz B, West SK, Rodriguez J, et al. Blindness and visual impairment and the problem of uncorrected refractive error in a Mexican-American Population: Proyecto VER. Invest Ophthalmol Vis Sci 2002; 4: 608-14.

[6] Quigley HA, West SK, Rodriguez J, et al. The prevalence of glaucoma in a population-based study of Hispanic subjects, proyecto ver. Arch Ophthalmol 2001; 119: 1819-26.

[7] Varma R, Ying-Lai M, Francis BA, et al. Prevalence of open-angle glaucoma and ocular hypertension in Latinos: the Los Angeles Latino eye study. Ophthalmology 2004; 111: 1439-48.

[8] AGIS Investigators. The relationship between control of intraocular pressure and visual field deterioration. Am J Ophthalmol 2000; 130: 429-40.

[9] Kass MA, Heuer DK, Higginbotham EJ, et al. The ocular hypertension treament study: a randomized trial determines that topical ocular hypertensive medication delays or prevents the onset of primary open-angle glaucoma. Arch Ophthalmol 2002; 120: 701-13.

[10] Lichter PR, Musch DC, Gillespie BW, et al. Interim clinical outcomes in the collaborative initial glaucoma treatment study comparing initial treatment randomized to medications or surgery. Ophthalmology 2001; 108: 1943-53.

[11] Heijl A, Leske MC, Bengtsson B, Hyman L, Bengtsson B, Hussein M. The early manifest trial group. Reduction in intraocular pressure and glaucoma progression: results from the early manifest glaucoma trial. Arch of Ophthalmol 2002; 120(10): 1268-79.

[12] Frisen L. High pass resolution and age related loss of visual pathway neurons. Acta Ophthalmol 1991; 69: 511-5. 